\title{
Impact of interaction paradigms on full-body interaction collocated experiences for promoting social initiation and collaboration
}

\author{
Ciera Crowell, Joan Mora-Guiard, and Narcis Pares \\ University Pompeu Fabra, Carrer Roc Boronat 138 \\ 08018 Barcelona, Spain \\ ciera.crowell@upf.edu, joan.mora@upf.edu, narcis.pares@upf.edu
}

\begin{abstract}
This article addresses the design and study of novel interaction settings and systems supporting collocated interaction. More specifically, we aim to analyze the implications of two different interaction approaches, namely first-person and third-person interaction paradigms, and the corresponding theoretical approaches when designing and developing collocated experiences for children with Autism Spectrum Disorders. This analysis will be supported by the outcomes of several examples of full-body interaction collocated experiences aimed towards promoting social initiation and collaboration. Moreover, we will present a series of goal-driven guidelines to consider when designing with various interaction paradigms. Finally, we will discuss future work to better understand the implications of constructing paradigms for intuitive use of these fullbody interaction systems.
\end{abstract}

Keywords: Autism Spectrum Disorders, ASD, collocation, embodied interaction, first-person interaction, third-person interaction, full-body virtual environments

\section{Introduction}

In the last decades there has been an increasing interest on including sensorimotor abilities in the interaction with computers. This can be seen both in Human Computer Interaction (HCI) research, with Krueger's Videoplace being one of the forerunners (Krueger, Gionfriddo, \& Hinrichsen, 1985), and in hardware development, with devices such as the Microsoft Kinect. In addition, interactive technologies specifically for children with autism, who have difficulties in sensory processing and motor gesticulation, can be developed to take advantage of these new variations from traditional interaction methods. In this diversification of HCI, we can find an increasingly important intersection between spatial interaction, bodily activity and physical interfaces. The paradigm which encapsulates this intersection is called Embodied Interaction.

Embodied interaction has unique properties when compared to other media paradigms, as users can take part in activities through direct physical manipulation of virtual content along with other users (Antle, 2013). Under the spectrum of Embodied Interaction exists full-body interaction, where users directly 
interact with the virtual environment through their gesticulations and bodily movements.

We understand that the full-body interaction paradigm encompasses all interactive media that sets the body and physical activity as the main means for communicating with the system. Full-body interaction technologies allow for a wide range of sensorimotor activity in forming the relation between the user and the virtual environment. Moreover full-body experiences can allow for faceto-face collaboration with other users during the interactive experience without the necessity of intermediary physical interfaces. Research has shown that fullbody interaction systems hold potential as intervention tools for individuals with social disabilities, such as those with Autism Spectrum Disorders (ASD) (MoraGuiard, Crowell, Pares, \& Heaton, 2016b). The current research is focused on exploring the potential of full-body interaction media for the design and development of collocated intervention tools for children with ASD. Also, we discuss the benefits of full-body interactive systems as free play environments for multiple users, their potential for exploratory and spontaneous collaborative play, which builds upon our previous work in social therapies for children with ASD.

Autism Spectrum Disorders are a collection of neurodevelopmental disorders affecting social and communication abilities and reciprocal interactions (Association, 2013). For individuals with ASD, forming and maintaining relationships with peers may be challenging due to deficits in social communication skills and understanding of non-verbal language. Restricted and repetitive behaviors, interests and activities can lead to motor skill impairments (MacDonald, Lord, \& Ulrich, 2014), passivity (Gabriels, Cuccaro, Hill, Ivers, \& Goldson, 2005), and sensitivity to sensory stimuli leading to over-reaction or under-reaction to stimulation (Lane, Young, Baker, \& Angley, 2010). Working with full-body interaction not only allows for users with ASD to practice sensorimotor skills, but also incorporates a larger range of communication, such as non-verbal communication, as systems detect expression through body language and proxemics. These non-verbal forms of communication are not usually accounted for in physical interfaces such as keyboards or controllers. We have also chosen full-body interaction as a medium for creating experiences where children with ASD can put into practice social skills with other peers based on its potential for natural and uninhibited interaction between collocated users.

Our research has overseen the development of three novel systems for users with ASD. "Picos Adventure" was an experience based on a third-person interaction paradigm, while "SIIMTA" and "Lands of Fog" were based on a firstperson interaction paradigm. We base our understanding of these two interaction paradigms as defined by Pares and Altimira (Parés \& Altimira, 2013). The two paradigms are defined by the relation between users and by the characteristics of the physical interface, which subsequently affect the user's experience of the virtual environment (VE).

In this article, we will first provide an overview of the role of interaction paradigms in constructing user understanding in collocated experiences. This discourse will be supported by the theoretical underpinnings of autism, interac- 
tion paradigms, and full-body interaction. Then, we will explain the aforementioned research work and the results obtained. We will analyze and discuss the implications of the interaction paradigm along with the results, helping other designers to understand the potentials of each interaction paradigm. Finally, we will present a series of suggestions for designing similar systems.

The main contributions of this article will include:

- Analyzing the implications of interaction paradigms in collocated environments.

- Discussing a series of characteristics related to the first and third-person interaction paradigms.

- Presenting a series of guidelines for designing new full-body interaction collocated experiences.

- Proposing solutions and future work for deeper understanding the implications of the interaction paradigm in constructing user experiences with children with ASD.

\section{Autism Spectrum Disorders}

Autism Spectrum Disorders (ASD) are neurodevelopmental disorders characterized by behavioral abnormalities in the domain of social communication, and also by restricted and repetitive behaviors, interests and activities (Association, 2013). ASD has an estimated prevalence of about $1.47 \%$ to $2,64 \%$ of the total population. Autism is more common in males than in females, occurring at a rate of 5/1, respectively (Kim et al., 2011; Christensen et al., 2016).

Social impairments pose a significant challenge for children with ASD. Nonverbal communicative interactions can be difficult to grasp for people with ASD (De Jaegher, 2013), as well as spontaneous social and play environments. As previously stated, full-body interactive media might have unique advantages over other interactive media as it allows for the development of non-intrusive technological solutions for developing social therapy tools for intervention through collocated environments.

These individuals might also face challenges creating and maintaining social relationships with colleagues. Therefore, it might be necessary to provide support solutions during unstructured social scenarios to counter social fragmentation and exclusion (Anderson, Locke, Kretzmann, Kasari, \& AIR-B Network, 2015). It is thus important to understand the advantages and limitations of interaction paradigms when working with full-body interaction technologies to properly use their potential as social intervention tools.

\subsection{Cognitive theories for ASD}

As ASD is a multifaceted condition, no single theory exists that accounts for the myriad of ages, intellects, and fluctuations that are encountered among individuals with ASD. However, cognitive theories for autism have emerged as an 
influential mark in the field. These theories include the theory of mind, executive dysfunction, and weak central coherence.

The theory of mind hypothesis holds that individuals with ASD are unable to take into account the mental states of themselves and others. This may explain difficulties in forming close social bonds and understanding non-literal or nonverbal language.

Executive function refers to a family of goal directed behaviors, such as planning, multi-tasking and inhibition. The executive function hypothesis for autism may account for difficulties in switching between tasks and controlling impulsive movements. In an experiment by Rajendran et al. (Rajendran et al., 2011), a running errands simulation tested multi-tasking and the point in which participants failed to effectively carry out tasks. It was found that inflexibility in planning and problems with prospective memory seemed to cause the most difficulties with multi-tasking among participants.

The weak central coherence theory aims to understand how individuals with ASD demonstrate significant difficulties comprehending overarching contexts, or "seeing the big picture". This can be observed in the tendency for children with ASD to possess pockets of knowledge and abilities, while still finding it challenging to live in the real world. In many instances, children with ASD will focus more on details and rigid routines, while missing out on larger concepts.

\subsection{Unique approaches to interaction}

Individuals with autism show a high prevalence of impairments in motor skills (Gillberg \& Kadesjö, 2003; Liu, 2013). In their research, MacDonald et al. (MacDonald et al., 2014) found a significant correlation between autism severity and deficiencies in fine and gross motor skills. Individuals with autism also show difficulties in sensory processing (Donnellan, Hill, \& Leary, 2013), which has led to an increased focus on developing therapies with augmented multimedia such as music and digital content. In addition, children with autism show dysfunctions related to proprioceptive systems, leading to disproportionate reactions to sensory input (Ayres \& Tickle, 1980).

Restricted and repetitive behaviors, interests and activities can lead to stereotypy or passivity (Gabriels et al., 2005). Stereotypies can be understood as repetitive, close to ritualistic, movements and utterances. Stereotypy behaviors may include from body-rocking to repetitive sensory self-stimulation. These characteristic behaviors can lead to an aggravation on the potential motor skills deficits characteristic of ASD (Johnson \& Myers, 2007). Nonetheless, there is evidence that stereotypies can be reduced through positive reinforcements to teach children to diversify their activity (Eason, White, \& Newsom, 1982). Systems based on tracking user's activity, such as full-body interaction, inherently have the possibility to track physical behavior and detect idiosyncratic behaviors, which could be reciprocally addressed by the virtual environment.

Finally, children with ASD tend to show unusual behaviors in imaginative and symbolic play when interacting with toys. They may persist on exploring objects, use them for self-stimulation, becoming entirely self-absorbed in 
these behaviors. Their approach to objects and toys tends to be an exploration through tasting and caressing (Rowland \& Schweigert, 2009; Williams, 2003), being extremely intimate and close to the objects. Therefore, while for them play remains an important activity in the development of symbolic and abstract thinking, children with ASD might approach playtime activities and toys in a different manner than neurotypical children. The use of full-body interaction environments in first-person interaction may be directed towards this population as it enables users to be "close" to the virtual environment and the objects within it.

\section{Third and First-person interaction paradigms}

In this section we will describe the first and third-person interaction paradigms based on the formal description done by Pares \& Altimira (Parés \& Altimira, 2013).

A paradigm is a theoretical framework with a solid and well recognized foundation or central viewpoint (Harrison, Tatar, \& Sengers, 2007). Building upon this, an interaction paradigm can be a model or pattern of human-computer interaction (HCI) that encompasses all aspects of interaction, including physical, virtual, perceptual, and cognitive channels (Heim, 2008).

Therefore, an interaction paradigm goes beyond the hardware configuration or physical interface, using these elements as a basis upon which to build the reciprocal interaction between the system and the user. As advances in physical interface design and ubiquitous computing allow users to break away from static working arrangements, intuitive interaction paradigms take precedent where users invert minimal time in training to work with these systems.

In full-body interaction systems, meaningful action takes place not only in the virtual world, as is the case in most classic VR systems, but also in the physical space, where the user's bodily activity and actions occur. On a physical level, this interaction space is meaningful as the space where the physical interfaces capture the users' movements. On a conceptual level, this movement space represents the real world counterpart which contextualizes the actions mirrored in the virtual space.

In our discussion of first and third-person paradigms of full-body interaction, we are referring to the perspective of the user in viewing their virtual counterpart: whether the user views an avatar, image or silhouette performing their mirrored actions (third-person paradigm), or whether the user views the effects of their actions without the presence of a visible counterpart in the virtual world (first-person paradigm). It is also important to note the difference between egocentric and allocentric perspectives in spatial cognition of virtual environments. An egocentric perspective refers to the understanding of virtual representations such as avatars, silhouettes, or mirrored images from the reference frame of the perceiver ("you") (Klatzky, 1998). Therefore the location is represented through the viewpoint of the object referent. An example would be giving directions with a map starting from one's present location. On the other hand, an allocentric 
(also known as exocentric) perspective refers to viewing a scenario from an objective frame of reference (he, she, it). That is, points and objects within the virtual environment are located independently of a viewers reference point. An example would be a map of an area produced by a mapmaker, where landmarks are depicted in static relation to one another and the nearby streets. In fullbody interactive systems where the user acts directly or indirectly through a virtual counterpart in the system, we can understand that the user is imputing an egocentric viewpoint on their virtual placeholder from both the first and third-person perspectives.

In full-body interaction systems the user's body has a sense of scale and direct relationship with the virtual space. Moreover, the digital representation of the user in the virtual world is a way of helping the user to understand this relationship. Furthermore, in collocated experiences, users not only have to account for the relations of their actions regarding the virtual experience, but also for the actions of their peers.

Full-body interactive systems can include a wide range of hardware arrangements, including large multi-touch surfaces and CAVE-like systems. They can also play on the user's senses, including tactile and auditory elements. In this discussion, we will use front or floor projected systems as example scenarios, as they provide two clear and distinct reference frames for system arrangement. In full-body interaction systems, the system can be based on tracking the user's movement by detecting the human body or by the use of a tracking marker, which is worn or held, and serves as the user's means to communicate with the system. Independently of the tracking solution in these systems there is always a logical representation of the user within the virtual environment, in first-person being invisible and in third-person being visible.

\subsection{Third-person paradigm}

In a third-person interaction paradigm, the user's physical embodiment and interactions are visually present in the virtual environment. This virtual counterpart is the digital means the user has for interacting with the virtual elements. The virtual representation can have many different shapes, one of them being the silhouette, or even the mirrored image, of the user. The user sees himself in the scenario as this virtual representation, and his actions are mapped onto this virtual counterpart Therefore, the user interacts indirectly with the virtual environment through a visual representation to interact with virtual objects.

This virtual counterpart has one primary purpose, which is to show the user the consequences of his actions in the virtual environment. Therefore, the player uses the visual feedback to understand how to position his body and gesticulate correspondingly to the virtual environment. In collocated full-body virtual environments, users have feedback of their peer's actions from both their physical actions and from their virtual representations. These virtual counterparts can

help users with autism to better perceive their peer's intentions, actions and position during the interactive experience. 
Users are linked from the physical world to the virtual world through mappings (Parés \& Parés, 2006). These are the configurations which translate user movement, speed, or scale into the virtual field of view. With an easily understood mapping between the user and the virtual environment, users construct a sense of control over the virtual surroundings. In camera-based setups such as the Kinect, when the camera is on the same side as the display, a distance may be necessary between the user and the visual display to assure that the user's whole body can be recorded. The camera records the user's bodily movements and maps them in real time onto a virtual representation, which the user sees within the virtual environment. In this case, where there is a significant distance between the user and the display, users may find difficulties in relating their actions to effects in the virtual environment. In this case, a visual representation is especially helpful to orient the user to the virtual effects of his actions. Therefore, a 3rd person representation might also be helpful for the user to understand the mapping between his movements and the display screen (see Figure 1).

The explicit visual representations inherent to the third-person paradigm can also be helpful in collocated environments to help people who have difficulties interpreting other's behavior, such as individuals with ASD, to better understand the intentions and actions of their peers during interaction. The virtual representation becomes an additional source of information from which to properly understand the intersubjective process happening during shared collocated play.

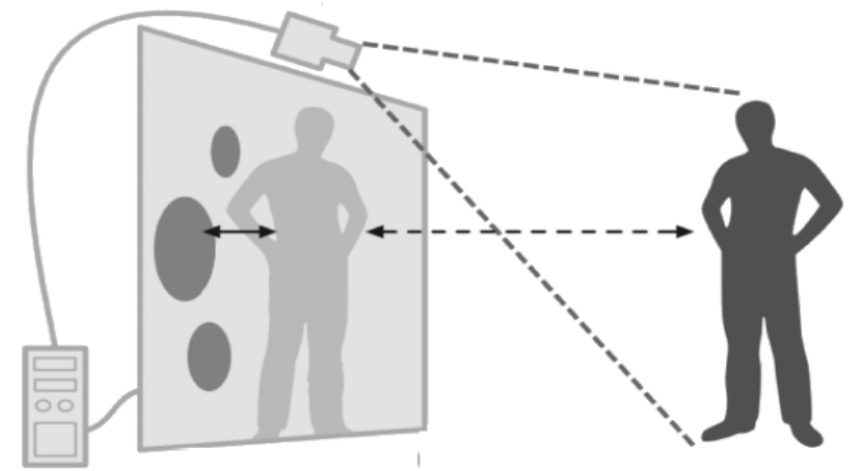

Fig. 1. A potential third-person paradigm physical configuration: A camera is placed in front of the user, so the user must stand at a significant distance from the screen in order for the whole body to be recorded (e.g. the use of the Microsoft Kinect with a regular TV).

It has been seen that individuals with ASD tend to embrace a predominantly egocentric perspective, viewing others based on their relationship to the self, with limited understanding of interpersonal relationships between others unrelated to 
the self (Frith \& De Vignemont, 2005). This difficulty in the ability to mentalize, also known as "mindblindness" (Baron-Cohen, 2008), has been well documented in children with ASD (Frith \& De Vignemont, 2005), and is seen as an extreme egocentricity and disinterest in the mental states of others, resulting in notable social impairments in relating to peers. Which effect does this have upon first versus third-person perspectives in full-body interaction? For these individuals, it is very difficult to switch between egocentric and allocentric perspectives (Frith \& De Vignemont, 2005). While playing a game through the perspective of an avatar, the user is still attributing an egocentric perspective onto this object or character. However, it is possible that autistic users are not aware that they are embracing an egocentric perspective, and rather think that their perspective is objective. In first and third-person perspectives of virtual reality, the difference should not matter to children with ASD, as they are both viewing the virtual agent as the self. The problem would come when they must impute mental states onto others (ToM), which requires activation of different neural correlates than that of the self. As is the current situation, switching from first person perspective to third-person perspective, subjects display a higher cognitive load resulting from transferring the egocentric point of reference from their own eyes, as is habitual, to the virtual avatar. In a test by Vogeley, et al (Vogeley et al., 2004a) the presence of this load was behaviorally observed based on the subject's higher reaction time and higher error rates, and biometrically evident based upon significant differences in neural activations in areas related to spatial cognition, as compared to first-person perspective (Vogeley et al., 2004a). In an experiment by Guido Peeters, et al. (Peeters, Grobben, Hendrickx, Van den Eede, \& Verlinden, 2003), is was seen that both children with ASD and typically developed controls preferred the use of self/other categorization in decision tasks, while only adults consistently used 3rd person categorization (allocentric). The researchers suggest that the development of egocentric categorization precedes that of allocentric, which might belong to higher cognitive processes.

\subsection{First-person paradigm}

In the first-person interaction paradigm, the user interacts directly with virtual content. In these systems, there is no visible counterpart of the user in the virtual environment. Rather, the user's movements have a direct impact in the virtual world, and the objects within it, and sees the effects of his movements represented in real-time (see Figure 2). Thus as ASD children tend to explore objects in a more intimate way than neurotypical people (Rowland \& Schweigert, 2009; Williams, 2003), an interaction paradigm that enables for the design of experiences where users have a direct interaction with the virtual elements might pose more suitable for this population.

In this paradigm, the system accounts for a virtual counterpart in the system which is invisible to the user. The user does not need to mentally map his movements onto a virtual counterpart or silhouette, but only needs to understand how his actions are mapped into the virtual world. In our experience, many users have a tendency to approach the visual display and touch the virtual elements, falsely 


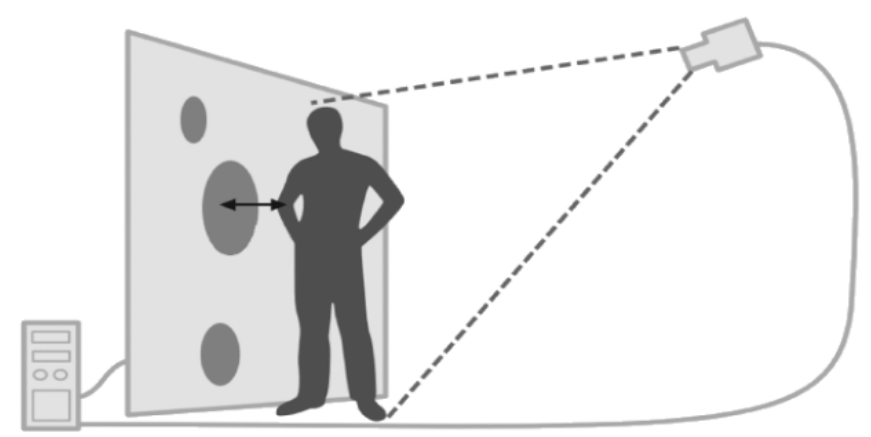

Fig. 2. One possible configuration for a first-person paradigm configuration which is camera-based. Rear placement of the tracking system allows for the user to be close to the display screen.

understanding that physical contact is necessary to integrate themselves into the virtual world. In the case of camera-based systems, this contact is not necessary, although it seems a natural practice for users to understand the reaction of the system to their movements. Therefore, it is important to allow for flexibility in the user's movement space, so they can undergo this process of learning to operate the system.

In first-person interactive environments, users do not need to impute their perspective on a third-person agent. This decreases the cognitive load of interacting with the system. However, systems must be carefully configured to match with the perceived mappings of the users so they can operate the system with ease.

In the first-person paradigm, these clear mappings are especially important as the user has no visual referent in the virtual space, and is only aware of the effects of their movements. To respond to user expectations, 1:1 mappings are useful between the user's physical movements and reactions from the virtual environment. However, this can be leveraged to affect user performance. In the case of children with ASD, who commonly demonstrate motor difficulties, the mappings of movement can be exaggerated to help the children hit virtual targets without strain. Conversely, a mapping which does not align with the user's expectations can cause confusion or frustration. Many people experience this disorienting frustration when switching to a different computer and the mouse pointer moves much faster or slower than expected. In first-person perspective, where these mappings might not be evident due to the lack of a visible virtual referent, designers must be mindful of the potential beneficial and harmful effects of tampering with unnatural mappings, and design the experience accordingly. The naturalization process might be hastened with introductory scenarios or consistent feedback. Nonetheless, framing the implications of first-person interaction in collocated experiences, users only have their peer's physical actions 
and the system's visual feedback as ways to understand other players' actions. This could lead to distributed focus both to the display and to their peers.

\section{$4 \quad$ Virtual Subjectiveness}

When developing interactive virtual experiences, in addition to defining the physical interface, designers must also consider the experience that will be modeled for the user. In addition to objects and environmental displays, each user will exist within the virtual environment as an actor, with a defined point of view from which they perceive the surroundings wherein (Ellis, 1991). As in real life, this point of view has a concrete set of parameters which contribute to the perception of the surroundings, defined by Pares \& Pares as "the Virtual Subjectiveness" (Parés \& Parés, 2006).

Each living being has a set of physical and psychological states which define his experience of the world in a subjective manner. Our capabilities, experiences, and attitudes contribute to how we perceive the world around us. As system designers, we have the ability to elect the attributes of the virtual perspectives which we construct, as users come into a virtual environment ready to receive the experience which has been designed for them. The virtual Subjectiveness model refers to the sum of these "filters" (i.e. the product of the interaction and content design decisions) which designers choose to compose the virtual point of view of the user.

Thus, deciding between implementing a system in a first-person or thirdperson paradigm will clearly affect how the user perceives the interactive experience. When using a first-person perspective, users have a "direct" manipulation of the virtual objects from the virtual environment. This configuration might make it easier for users to understand their manipulation capabilities of the virtual environment through their physical actions. Nonetheless, as this approach tends to make users have to be closer to the displayed content, it might also create difficulties for users to perceive the whole virtual environment. In contrast, third-person paradigm might help users to get a better perception of the whole virtual environment thanks to an easier egocentric perspective, due to their body being virtually represented in the digital environment. Nonetheless, approaching the third-person paradigm through mirroring user's captured image, might bring cognitive burdens as users must adopt their playing style to the imposed mapping. As previously discussed, interaction paradigms can deeply affect also how users will perceive their peers actions in collocated experiences, as digital content might help better understand others' intentions in a third-person paradigm, but could also affect how much attention is put on the physical world interactions. Thus, the interaction paradigm implemented will also affect how users socialize, communicate and collaborate.

With the purpose of creating transparent or intuitive interfaces, designers aim for a very short adaptation stage where users can start using systems in an natural manner. This has led to the idea that an intuitive interface can be neutral, without biasing the user through predefined filters. However, these in- 
terfaces are never neutral. Although a virtual experience might include all the same sequences and measures as a real life action (e.g. landing a plane in a flight simulator), a mediation remains constructed by the designers, in the viewing angle, resolution, degrees of freedom, and user positioning. These configurations provide a specific way of understanding the experience. Therefore, maintaining awareness of the virtual subjectivity is how designers can understand the user within the experience. Designers have the ability to leverage this potential to their advantage, bringing it to the foreground of the virtual experience. If correctly implemented, this tool will not only contribute to the user's phenomenological experience, but also lead the user to understand how he can interpret his influence and control over the states of the system.

\section{Embodied interaction and cognition}

Independently of which interaction paradigm is adopted by designers of full-body interaction systems, users will interact with the virtual environment through their bodily activity. Users' perception of the interactive system is based on their active participation within it, giving to the body a key role in the interactive experience. Also, as recent theories on cognition pose the body as a key component in psychological processes such as learning, it is important to analyze these paradigms to understand the importance of the body and the subjective experience related to its activity.

Embodied cognition theories state that cognition is mediated by the human body and its place within the surroundings (Borghi \& Cimatti, 2010). Phenomenology philosophy stresses the triadic relationship between the world, the body's place within it and the time-constrained experience, necessary for the construction and development of cognition (Cerbone, 2006). Constructivist Theory proposes that cognitive development is based on the "detachment" of knowledge from the world of concrete objects to the world of symbolic objects, knowledge emerging as a result of activity within the world (Ackermann, 2004). Finally, Activity Theory states that cognitive processes also emerge from the activity of humans in the social environment and the artifacts within it, meaning inter-subjectivity is necessary for the development of cognitive processes such as learning (Wertsch, 1985).

We can see from the different disciplines that the body is seen as a key mediator of cognitive processes. We can also understand that the active participation of people with other subjects and objects in the environment is necessary for the development of knowledge. For Hanne De Jaegher, allowing to experience learning activities where there is a freedom for repetitive interests and behaviors might be motivating for children with ASD, fostering the learning outcomes of the experience, proper behaviors and social interactions when working with peers (De Jaegher, 2013).

In the last decades there has been a strong evolution towards novel ways of interaction between people and computers. In 1990 Grudin proposed that the history of computing is that of the computer reaching out (Grudin, 1990), mean- 
ing that computers would increase involvement not only from the input of simple devices, but also from the user and their surroundings. Nowadays, more natural interactions are being explored, where users can have a more active and participative status in the experience by allowing for a wider range of sensorimotor activity to take place during the interaction process.

In his book "Where the Action is" (Dourish, 2004), Dourish derives the concept of "Embodied Interaction" from phenomenology. One of the major lessons he draws is that embodiment encompasses the relationship between action and meaning (Dourish, 2004, p. 126). Embodiment is not just about being physically present in the world, but also embracing a "participative status" within it. The new myriad of technologies available allow for the development of novel systems which allow users' motor abilities to be involved in HCI, thus offering systems where the user is increasingly participating in the virtual world. For Lindblom and Alenljung approaching the design of technology through an embodied interaction lens is pivotal for designing experiences that take into account the social nature of human beings (Lindblom \& Alenljung, 2015). For Hartson and Pila (Hartson, Rex, 2012) embodied interaction helps to move the interaction from the display to the real world, thus emphasizing the importance of both motor and social actions taking into account in the real world. Moreover, embodied interaction seems to have unique potential for developing collocated systems which allow for direct natural manipulation of the virtual environment (Antle, 2013; Dillenbourg \& Evans, 2011).

Drawing from this, full-body interactive environments allow for the body and gestures to become the focus, as participants may operate the system through natural kinesthetic movements (Grandhi, Joue, \& Mittelberg, 2011; Nielsen, Störring, Moeslund, \& Granum, 2004) and light physical interfaces. In this respect, we believe that full-body interactive environments which allow for the use of body language and communication gestures could be particularly useful for facilitating social understanding, making this kind of media more suitable for developing systems for children with ASD. Research has shown that this interaction paradigm approach is successful in fostering user's engagement (Bianchi-Berthouze, Kim, \& Patel, 2007) and also for learning (Antle, Corness, \& Droumeva, 2009; Howison, Trninic, Reinholz, \& Abrahamson, 2011).

Full-body interactive environments based on user states can be advantageous for the learning of concepts (Revelle, 2013). This was demonstrated in an experiment by Benson and Uzgaris, where babies who were allowed to crawl through an environment found hidden objects easier than babies who had been carried through (Benson \& Užgiris, 1985). This shows how first-person exploration, not to be confused with the first-person interaction paradigm, of an environment leads to mental model construction (Bartoli, Corradi, Garzotto, \& Valoriani, 2013). Also, the framework of Embodied Facilitation describes how the layout of material objects and space relates to group behavior (Hornecker \& Buur, 2006). This theme is important when designing play experiences, as providing feedback to physical activity can be implemented in group settings to stimulate physical play (Bekker \& Sturm, 2009). 
As individuals with ASD commonly have difficulties with motor skills (MacDonald et al., 2014), full-body virtual environments allow a freedom of movement beyond the traditional mouse and screen setup (Chen, 2012). Also, collocated full-body experiences allow for face-to-face collaboration with other users, which has been seen to foster social behavior (Mora-Guiard, Crowell, Pares, \& Heaton, 2016a). Therefore, users can interact with each other without a technological medium mediating the communication channel; technology serving as the scaffold and motivator of communication.

\section{Related work}

Previous work on full-body interaction systems for both individuals with ASD and typically developing children has addressed the need to scaffold social and collaborative play in engaging environments.

"Lightpools" ("El Ball del Fanalet" in Catalan) (Hoberman, Pares, \& Pares, 1999) was a full-body interaction collocated artistic installation for the general public. This artistic project was developed for exploring the potentials of fullbody interaction technologies for developing multi-user experiences. Up to four users at a time explored a virtual environment through the use of hand-held pointers shaped as traditional Catalan paper lanterns, called fanalets. While exploring, users could "feed" basic virtual objects, called proto-objects, with light to obtain more complex abstract objects, which later could be trained to perform simple or complex choreographies between different users (see Figure 3). Although users had a handheld pointer, their actions were directly mapped into the virtual environment, and the virtual objects would react in a 1:1 mapping to user's movement and position of their paper lantern. Thus, the system was based on a first-person paradigm used for a floor-projected system, through the use of handhelds as a means of interaction. The first-person approacj also seemed to ease the way users could collaborate, as users could build between their objects different choreographies by moving their lanterns close, without having to deal with mediating virtual counterparts.

The MEDIATE project (Parés, Masri, van Wolferen, \& Creed, n.d.) was the first full-body interactive system designed for individuals with ASD. MEDIATE was specifically designed "for children with autism to have fun and have the chance to play, explore, and be creative in a controllable and safe space" (Parés et al., n.d.). The project was an interactive multi-sensory space with different sensors and actuators to work with different types of stimuli. Two of the installation walls were real-time interactive screens which reacted to user's position, movement and touch. The virtual environments were composed by a series of aligned squares which would grow as users got closer to the screen, but only where their silhouette would be projected. If the user got close enough, the squares would form the silhouette of the user in a low-resolution fashion. Moreover, if the user touched the screen, a ripple effect would appear originating from the touch (see Figure 4). All these visual feedbacks were designed to help the user understand their own physical shape and physical actions. MEDIATE was 


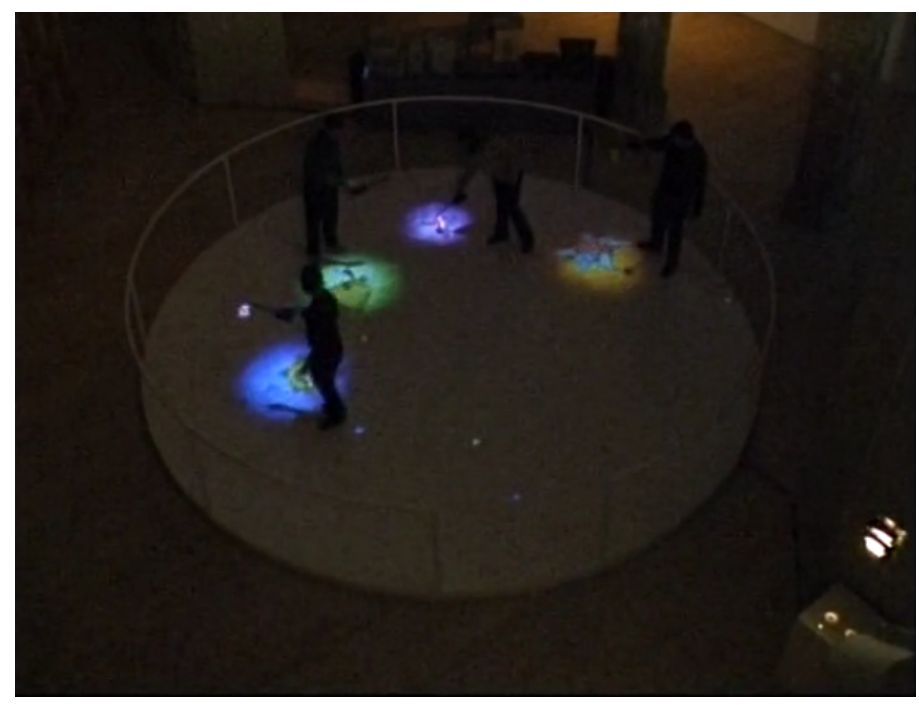

Fig. 3. Top view of the Lightpools collocated experience. In the image four users are leading the virtual objects, which follow the paper lanterns around the environment.

a first person experience, as users were not explicitly represented visually in the virtual environment for users to understand their relation with virtual content. Although the combination of the squares would take at the end the shape of the user, this was a consequence of user's movement, rather than a continuous representation of them. The first person approach was beneficial for helping users be self-aware on how their physical actions affected the digital environment, as no external representation, to which users would have had to mentally map their actions, was mediating the interactions between digital world and physical world.

In 2007 Wendy Keay-Bright developed a playfully exploratory system for ASD children in which they could easily explore different "magical" interactions without previous knowledge of the technology (Keay-Bright, 2007). ReacTickles aimed to explore the possibilities of interactive virtual environments to foster children opportunities for expressing themselves and foster immersion in the learning processes during playful intervention. The system was based on a series of playful experiences based on basic feedbacks such as particles, and was implemented for being used in smart whiteboards of schools. The system tracked user's silhouette, which was used to interact with the virtual environment. The system was also designed in a first person interaction paradigm, as MEDIATE was, as users were not virtually represented in the virtual environment. Given this design, the system was later easily transferred to tablet devices. The first person approach could have proven beneficial for users to quickly adopt the interactive systems and understand the relation of their actions with virtual content reactions. Through our work, we will discuss how first person might be helpful 


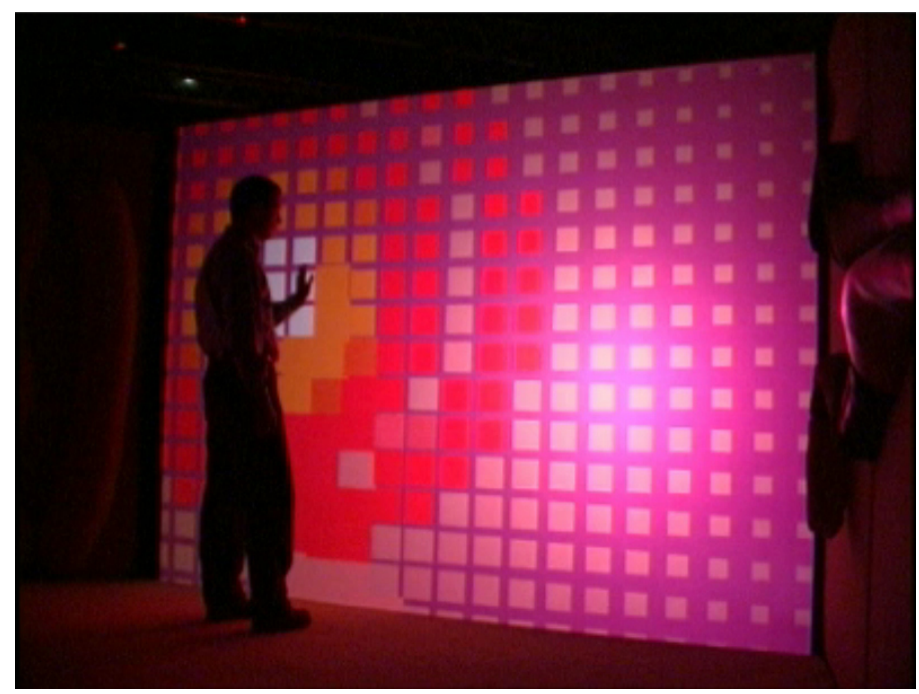

Fig. 4. Vertical full-body interaction screens that composed 2 out of 5 multi-sensorial walls of the MEDIATE project. Upon touch, the system displays a ripple effect originating from the user's hand.

for users to understand the relationship between their actions in the physical space and the reactions of the virtual content.

"The Pictogram Room" is a full-body interaction videogame in which pictograms were superimposed over the bodies of children with ASD. The purpose of the project was to help understand the relation between iconic pictograms and the human body. The project was aimed at supporting communication, joint attention and imitation therapy for social behavior learning in individuals with ASD (Casas, Herrera, Coma, \& Fernandez, 2012). In this project an augmented reality approach was taken to superpose pictograms to the body gesticulations of children to easily communicate the relation between drawings and body. During the experience, the application would be displaying the user's mirrored image, helping them understand the pictograms represented in relation to their own body. During collocated scenarios, both users could see their peer on the screen, which helped to perceive both their own and their peer postures and overlaid pictograms by looking at the display. Thus, the experience was designed as a third person interactive experience, where the user's image served as a tool to aid in understanding actions and iconic representations within the virtual environment.

SensoryPaint (Ringland et al., 2014) was a large multimodal system where users interacted through bodily movements to virtually paint on a large display. The system integrated handheld tangible objects in the form of rubber balls to serve as the paint brushes. This project addressed the difficulties in sensory processing which accompany the autistic condition, as well as extended engagement in sensory therapy sessions. The system was designed for individual use, but re- 
searchers found that participants showed positive social behaviors from working with the system collaboratively. As the user interacts with the system though a full-size silhouette which is projected into the virtual environment, this system represents a third person perspective experience.

BendableSound (Ortega, Cibrian, \& Tentori, 2015) was an interactive surface project designed for children with autism to experience multisensory development within a free play environment. The project consisted of a fabric surface upon which digital figures appeared. Users could interact with the figures through various gestures, including tapping and grasping the fabric. Different gestures yielded various musical responses from the system, so users could practice motor movements through interacting in a first person format. Recent versions of the system have implemented both goal-oriented and free play scenarios, as well as greater sound variability according to the intensity and location of the touch (Vazquez, Cardenas, Cibrian, \& Tentori, 2016).

Another full-body interactive project for acquiring social skills was "The Echoes Project" (Porayska-Pomsta et al., 2011). The scope of this project included developing a virtual environment which would encourage children with ASD between the ages of 5 and 7 to explore and acquire social interaction skills. The system was based on a virtual environment where a digital avatar was able to interact with the children by asking their help in a series of tasks, and displaying joint-attention behaviors. The system used an artificial vision subsystem to interact with the children and track children's focus towards the virtual objects. This way the system allowed for activities where joint attention, such as pointing, could be practiced. This is a non-paradigmatic case, as the system was based on a multi-touch TV, but was designed as a first person interaction experience. Users had to touch the different elements the virtual avatar asked them, and their touch actions were mapped 1:1 to content movement.

\section{Present work}

In this section we will introduce our experience designing full-body collocated interaction systems for children with ASD to motivate participation and social behaviors, including collaboration. We will present three systems which we have developed to explore the potential of full-body interaction to develop intervention tools for children with ASD, first explaining the motivation behind their design and goals, and later explaining the technology used, the interaction design and the interaction paradigm in use. We will categorize the systems based on the interaction paradigm we used. First, we will introduce Pico's Adventure, a virtual experience based on the Microsoft Kinect camera to promote social initiation behaviors between collocated users. Then, we will present SIIMTA, a single user music-therapy system which inspired Lands of Fog, a large floor-projected multiuser collocated interactive system to promote positive social and collaborative behaviors. 


\subsection{Third-person paradigm}

Pico's Adventure This project was a full-body interaction videogame developed for the M4ALL European project in 2012 (?, ?). The goal of the project was to develop a series of motion-based playful experiences for children with intellectual and motor disabilities. One of the conditions of the project was to use affordable and widespread interactive technology. Therefore, the system used a Microsoft Kinect to detect user movements and a large TV screen as a display. For the design and evaluation of this system we partnered with a team of psychologists specialized in children with ASD.

During the first levels, parents were asked to join in the game, forming part of the collocated multi-user dynamic. Having the children play the videogame together with a partner was meant to motivate awareness of social initiation behaviors, promoting behaviors such as approaching others, starting social communication and producing verbal or gestural behaviors for communication goals. Finally, during the last session, two users with ASD were collocated together, side by side in front of the visualization screen.

For Pico's Adventure we adopted the third-person point of view paradigm, partially imposed by the technology we used. As we wanted children to put into practice specific social skills, such as stimuli discrimination and joint attention, the technology chosen to implement the video game was the Microsoft Kinect, a camera that allows for gesture, position and movement tracking. This technological approach places the user at a minimum of 2 meters away from the display. Thus, the use of this camera, which was placed in front of the TV and facing the user, supported the adoption of the third-person interaction paradigm, digitally representing the user in the virtual environment. We did this by capturing the user's image and rendering it in the virtual environment as if looking into a mirror (see Figure 5).

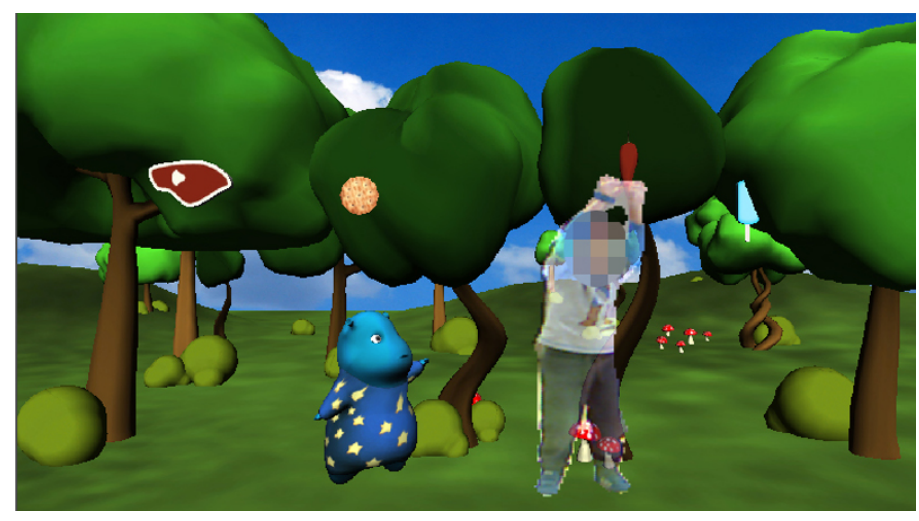

Fig. 5. Image of a child giving a virtual element to the projected character. In the image it is possible to be see how the third-person interaction paradigm was applied through mirroring user's captured image. 
In the virtual environment, users could see their own reflection beside that of their partner. This gave the impression that the two players were together in the virtual world, and they naturally interacted with their partner in joint actions such as holding hands in order to overcome challenges in the game narrative.

Integrating the users virtual representation in a "mirror fashion in the displayed scenario was also decided for helping users understand their relation with the virtual environment through an egocentric perspective. When practicing social skills, children with ASD were able to see their own image doing the task, reinforcing understanding of their actions. A virtual representation of the user's body within the VE was also necessary as part of the interaction feedback, which resulted in an indirect interaction with the virtual objects (Parés \& Altimira, 2013). With this configuration users have to go through a first process of understanding the mapping between their actions and their virtual representation, and their relation with the virtual environment.

We implemented in the system behaviors such as:

1. Conventional gestures: promote the use of gestures based on socio-cultural conventions (i.e: Say bye with the hand).

2. Reciprocity (Turn-taking): define a situation that requires users to respect turns during cooperative games or activities.

3. Initiation and answer in Joint Attention: promote behaviors that requires an attempt by the child to call the attention of the adult toward an object that nobody is touching (i.e. pointing to something).

4. Cooperation: define situation that requires the child to build/do something together with somebody else

We divided the different behaviors children had to put into practice around a story about helping a lost alien return to its home planet. All behaviors were distributed between four different levels, where children would put into practice required behaviors in an incremental difficulty. As some behaviors are based on selecting or pointing to virtual objects, visual feedback was added to different play scenarios. This visual feedback was designed both to help users better understand the mapping of their actions through their virtual representation. Also, visual reinforcements could help them apply desired behaviors as cognitive offloaders. One example was that when children had to point to objects, a "magic laser", given by the alien to the users, would be casted from the user's hands (see Figure 6). This way, it was easier for children with ASD to understand and control the direction of their pointing in the third-person paradigm.

\subsection{First-person paradigm}

SIIMTA Prior to Pico's Adventure, we developed the SIIMTA music-therapy tool in 2009. SIIMTA (the acronym for "Real-Time Full-Body Interaction System and Music Therapy for people with disabilities or disorders such as Autism" in Catalan) is a full-body interaction environment music-therapy tool for children with low functioning ASD. The goal of the project was to develop a solution 


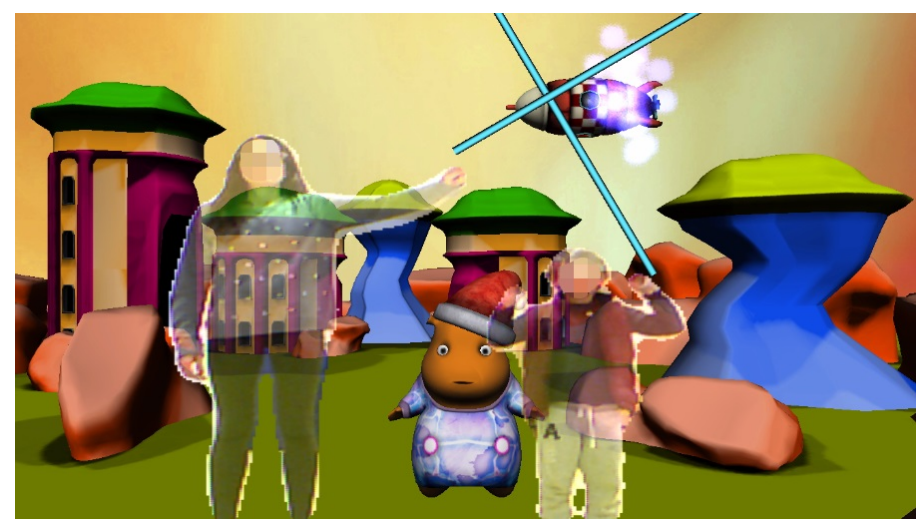

Fig. 6. Collocated players pointing to a virtual element. From each player's right hand, a visual feedback is projected in the direction that the user indicates.

for motivating user engagement and activity during music-therapy sessions. The project's music-therapists communicated the idea of fostering social attitudes while playing with caregivers and partners. The project was deeply inspired by the MEDIATE project, adopting the idea of developing an experience with serendipitous reactions of the virtual content to user's actions, through simple but effective interaction mechanics. In this project, a therapist or caregiver was present during experimental sessions, and served as the counterpart of social behaviors in the form of comments and observations. This dynamic sparked the idea of collocated interaction in posterior projects, as we decided that collocating the counterpart within the interaction space would lead to dynamic and actively engagement in gameplay.

SIIMTA was designed as a first-person interaction paradigm installation, utilizing MEDIATE's full-body interactive screens technology and design principles. The positioning of users closely in front of an interactive screen allowed for a 1:1 relation of users with the virtual environment, translated into what can be understood as a direct interaction of their body with the virtual objects. Creating a system where users have a 1:1 relation to the virtual environment relieves the process of understanding mappings from the interaction space to the virtual projection, allowing for a more natural and easy to grasp experience. This can be beneficial for children with low functioning ASD, which tend to have more severe sensorimotor challenges. We decided with the foundation therapists that the users had to be motivated and engaged because of the interaction rather than the application content. The system had to react to the users' movements promoting the user's curiosity to engage with the system. This decision was also taken from the previous knowledge acquired with the design of MEDIATE: "Therefore [...] the interaction had to be based on very clear action-reaction situations and probably rely on full-body interaction" (Parés et al., n.d., p.3).

Interaction design for SIIMTA was based on the "sound hysteresis" concept proposed by the foundation, which was a concept that also drove their music- 
therapy exercises. In this project, the concept of sound hysteresis was one of associating movement direction and sound pitch. Therefore, we created a design based on a sound space where major and minor chords would match to ascending and descending movements respectively. In addition, virtual particles would ascend and descend according to the fluctuations in sound and user movement. As the virtual particles were divided into columns, users could easily control the virtual objects as they gravitated up and down the vertical axis.

Moreover, when falling, particles would rest around the user's silhouette, helping them better understand the mapping between their physical volume and the digital embodiment (see Figure 7). Despite the fact that, on some occasions, accumulated particles over user's silhouette would partially draw the physical shape of the user, this cannot be understood as a first-person paradigm. In this case, particles as virtual elements were reacting to the user's body. The user's body silhouette was partially drawn, not as a means of representation, but as a reaction to its movement and position.

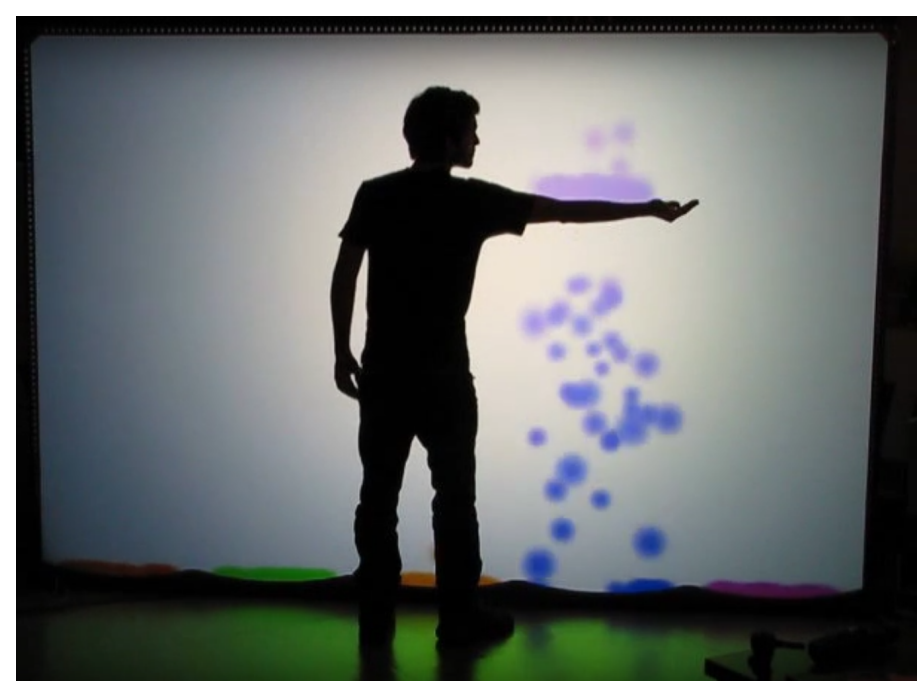

Fig. 7. A user holds falling blue particles with his right hand. Particles which collide with the user's arm stand still, giving the sensation of "physically" colliding with the projected volume of the user.

Lands of Fog In 2014, Lands of Fog began as a first-person interaction project based upon observations from the aforementioned two projects. In this case, rather than using a vertical screen as in SIIMTA, we used a large-scale floor projection. In this format, users could wander through the virtual environment in a 1:1 mapping with the virtual content, based on the project predecessor Lightpools (Hoberman et al., 1999). We believed that the use of a non-vertical 
screen could foster better social engagement than Pico's Adventure as it would be more natural for users to look first at the floor and then to their colleagues, rather than staying attuned to a TV screen. This new first-person paradigm system was Lands of Fog, which was created for the IN-AUTIS-TIC research project funded by the RecerCaixa 2013 grants program. The goal of the research project was the design and development of a multi-user collocated full-body interaction experience to promote social and collaborative behaviors. For results of the experimental trials, please see (Mora-Guiard et al., 2016b)

The project consisted of a large, circular floor-projected virtual environment where children with ASD would actively explore with a partner and play with interactive features such as mythical creatures and magical objects. The firstperson approach to the physical interface allowed users to be in direct contact with the display, without the necessity of a minimum distance and virtual representation of the user. In Lands of Fog users interact with the virtual environment through the use of a handheld pointer in the form of a butterfly net fitted with color markers to aid the tracking system. There was no visual counterpart to the handheld pointer, hence the first-person interaction paradigm, but the user's movement was clearly visible due to visual feedback based on a deep fog, which covers all the virtual environment, only moving aside at the location of the butterfly nets (see Figure 8).

This practice of restricting the view to reveal only a small section of the scenario is known as a "peephole", a design strategy suggested by Dalsgaard and Dindler (Dalsgaard \& Dindler, 2014), which has been shown to be a good practice for peaking curiosity and promoting exploration through discovery of the unknown. Additionally, the use of a handheld pointer was another design choice inherited from the Lightpools installation, as it was see that handheld pointers could be helpful as cognitive offloaders for users while interacting with the virtual environment.

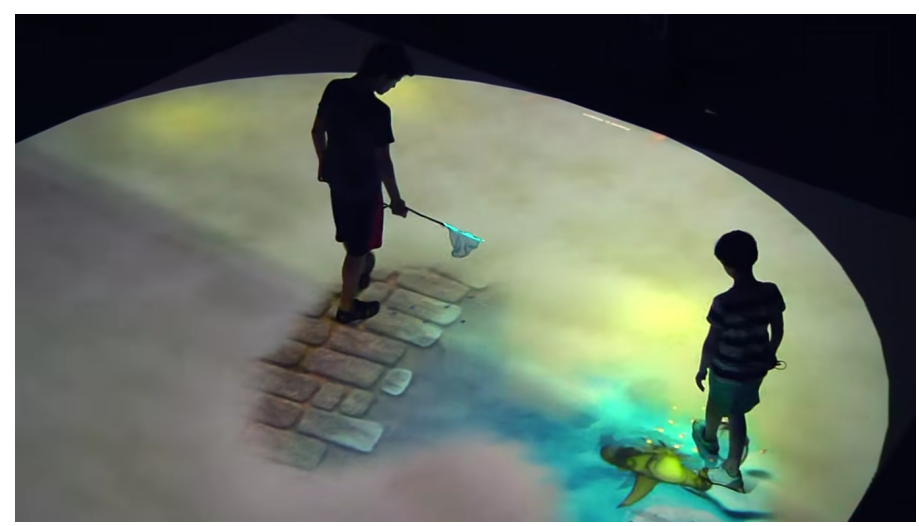

Fig. 8. A Lands of Fog with two players collocated in the scenario. Player blue, in the middle of the image, is exploring the environment through the fog with his blue butterfly net. In the right bottom of the image, the partner has already found a creature. 
As users explored, they obtained sophisticated virtual elements, such as autonomous creatures, which helped users perceive their invisible counterpart in the system, as these creatures followed the user by going where their butterfly net was placed (see Figure 9). For a detailed description of the game refer to (Mora-Guiard et al., 2016a). The decision to collocate two users in the system was made so that users would have a chance to practice social behaviors with another during the course of the game. The system was formatted for two players: one with ASD and one typically developed. We felt as if the addition of a TD playing partner would encourage inclusive attitudes towards involving children with Autism in play activities during and after game sessions. As many ASD social training systems are theory based and do not offer the chance to play conjointly in a collaborative atmosphere with a peer, we thought it would be important to provide this opportunity to ASD players in a safe and comfortable environment.

The typically developed playing partners were not trained in expected social interaction behaviors, but were encouraged to play in a natural manner. This protocol was decided in order to create an authentic game experience for both players, mimicking the spontaneity of classroom or playground environments. The game was configured to physically bring players together through the behaviors of the virtual elements, which detecting passive behaviors in children, would move in an interesting manner to bring players towards their partner.

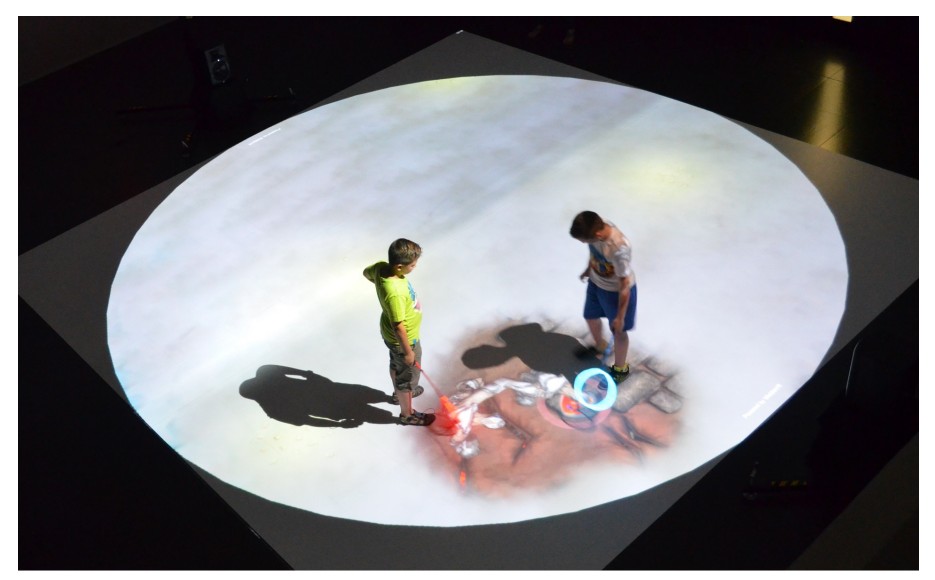

Fig. 9. Two players in the full-body collocated installation Lands of Fog experience combining their creatures to interact collaboratively with a virtual element.

\section{Discussion}

During the evaluation of the projects, we observed differences in user understanding and behavior alongside their partner as a result of the corresponding 
interaction paradigms. This section will analyze our findings during the evaluation of the systems related to the interaction paradigm in use. The discussion of the results obtained by each intervention tool is out of the scope of this article. We will limit our discussion to referencing obervations and outcomes obtained in relation with interaction paradigms.

\subsection{Third-person paradigm}

Account for added cognitive load During the user trials of Picos Adventure, we observed an initial difficulty in user understanding and handling of the physical interface. We believe these challenges arose given that the third-person paradigm caused an indirect interaction with the virtual objects and the partner's virtual representation, as the user interacted in the physical space through his own virtual image. This is consistent with findings from other 3rd person interfaces which revealed an extra cognitive load on users during the transfer of "self" mentalization from first person to third person point of view (Vogeley et al., 2004b).

Work alongside natural tendencies In Pico's Adventure, we also observed a common misunderstanding between users in their natural tendency to move towards the display and attempt to use it as a multi-touch device. This was to be expected, given the rise of the use of these devices in the last years, including for individuals with developmental disabilities (Stephenson \& Limbrick, 2015). This behavior was especially common when children tried to get close to the screen and touch the virtual agent, their peer's reflection, or the interactive elements. This created a conflict for the tracking system, as it was configured for users to be at a minimum distance of 2 meters from the tracking camera.

When designing digital content, it is important to take into account the affordances and physical constraints of the technology in use. As this physical interface is configured for users to remain at a distance, it breaks user expectations of physical contact with the content. In situations like this with strict user positioning requirements, forcing users to stand away from the screen might increase the difficulty to properly perceive digital content and feedback, thus designers must also take into account how to not block natural tendencies and rather work with them.

Personalization through identifying user preferences Moreover, the thirdperson paradigm yielded another interesting scenario. During the participatory design sessions, a child with autism refused to play because his image was being displayed on the screen by the third-person approach, similar to seeing his reflection in a mirror. His parents also reported that their child did not like to see himself reflected in mirrors. With this sensitivity to to sensory stimuli in mind, the game was adapted so it would be possible to configure whether the virtual representation of the user was their captured image or their silhouette filled with a gray color. Thus, the child was able to collaborate through all of the participatory design and pilot trial sessions. 
Maintain awareness of physical constraints While video coding the sessions, we searched for integration of social behaviors between the collocated peers, understood as combining gestures with speech and gaze during communication. We observed that although the children showed significantly high levels of social initiation behaviors, most of them were not accompanied by eye contact, a finding coherent with autism literature related to social initiations (Koegel \& Frea, 1993). These results also raise relevant research questions related to the physical constraints of the user interface in the collocated experience. As most social interactions lacked physical gaze towards the partner, we believe that the side-to-side placement of users in front of the TV could have especially hindered eye contact between users during social contact.

The physical configuration with a front placed Kinect could have played a role in limiting the amount of gaze physically directed towards the other player, as children were required to be one next each other and looking at the TV. Moreover as users could "see" their peer on screen, it is possible that they used their peer's virtual counterpart, from the third-person configuration, as an objective of the directed gaze. As gaze integration is viewed as pivotal to the proper development of social communication behaviors (Frischen, Bayliss, \& Tipper, 2007), it is important to understand how the physical interface and the interaction paradigm will influence behaviors when designing systems to foster socialization. In our case, having chosen an interaction paradigm which could hinder gaze integration in social initiations might have resulted in a lower complexity of socialization.

However, it might also be worthwhile to analyze the goals of the target interface, as in some cases gaze might be redundant or even harmful during collaborative tasks (for example, sustained eye contact while co-piloting an airplane). This point calls into question the use of integrated social behaviors as a one-sizefits-all determinant of success in collaborative tasks for children with autism.

\subsection{First-person paradigm}

Use 1:1 mappings In SIIMTA, the first person interaction paradigm seemed more adequate for the target population, as children with low functioning ASD tend to show increased difficulties with motor skills. We believe that the firstperson paradigm adopted in SIIMTA helped children with ASD quickly adapt to the new experience, which resulted in a $0 \%$ dropout of users related to rejection during the experimental assessment. Designing the system with a 1:1 mapping and natural interaction could have helped them to easily understand the system dynamics.

In the Lands of Fog trials, although users were not explained the interaction principles, we observed a fast and seemingly intuitive adoption of the game's interaction mechanics. We attribute this to the natural familiarity of exploring through an unfamiliar setting in first-person view, without the cognitive load of mapping movements onto a third-person representation. This setting could be understood as a Mixed Reality experience, with a direct interaction between the users movements and the virtual objects. 
Consider the influence of hardware arrangements In virtual environments where users must be far away from the corresponding digital content, we have seen that simple mappings or third person counterparts can help users correlate their physical movements with the virtual response in the system. However, designers must also consider how having a third person counterpart present in the system can distract user's attention when working in collocated environments with other users.

In Lands of Fog, we wanted the multiple players to experience physical exploration of the system together, so our selection of a system projected onto the floor was meant to allow for physical flexibility. The first person paradigm of the system allowed children to feel like they were exploring the system through their own eyes, a directness of interaction which we felt was appropriate for an exploratory multi-user system meant for engaging users and promoting social behaviors.

Moreover, the amount of integrated socializations in Lands of Fog was higher than in Picos Adventure. As the focal point of Lands of Fog was a large floor projection, there was a much greater freedom of gaze afforded to users, as compared to the small frontal screen used in Pico's Adventure. This also encouraged users to work with each other, as their collaborations took place at eye level.

Allow for multisensory approaches The use of a vertical large surface in SIIMTA, plus the interaction paradigm adopted which allowed users to be physically close to the display, yielded interesting observations. The screen used during the experiments was based on an elastic fabric specifically configured for retroprojection. We observed during the initial experimental sessions that a high amount of children got close to the screen and experimented with the screen by caressing or pushing it. This is in consonance with how children with ASD typically explore objects and toys through caressing, sometimes showing additional inconsistencies in sensory processing. As this project was in first person perspective, no minimum distance was required in the interaction space, therefore users had the impression of physically manipulating the virtual elements to generate a system response. This was not the intended interaction as the system responded to movement and not touch but nonetheless seemed to help children with ASD become acquainted with the system. Discussion with the on-site psychologist and caregiver during experiments revealed that these behaviors seemed to be a sensory approach for modulating their excitement with a new system and scenario. Thus, with SIIMTA the interaction paradigm not only affected users' subjective visual perception of the experience, but also their physical perception of the system.

Leveraging this adherence to multisensory stimuli could be useful for creating first person interactive displays and surfaces specifically for children with autism, such as BendableSound (Ortega et al., 2015). Therefore, for future first person systems it might be interesting to pay special attention to the materials used and their sensory affordances. 
Borrow cultural symbols for natural interaction Another difference between the systems is that in Lands of Fog, a handheld pointer was used to interact with the environment through capturing virtual fireflies. The idea of using a pointer was also inherited from the Lightpools system (Hoberman et al., 1999). The pointer, with the shape of a butterfly net, was used for both the tracking of user's actions and also as a cognitive offloader to draw attention to the task at hand. As the floor projected configuration meant interaction would be happening below users, we assumed that having a pointer would help users to better focus on the action and move gameplay elements freely around their body and around the interaction space of their peer.

Having visual cues in real-time, such as the peephole in the fog, helped users quickly understand the relation of their physical actions with the virtual reactions, similar to the simplicity of SIIMTA, even though interaction was mediated by an external handheld pointer. Moreover, using a pointer device for interaction with the shape of a butterfly net helped children with ASD understand how to interact with the world, using the cultural affordance of catching which was readily understood with the net (Horn, 2013).

It was noted that, when users were carrying the pointer in their hand and exploring the virtual environment projected below, this resulted in a distraction from eye contact with other users in the virtual scenario. Future work should aim to engage users in the virtual experience of floor projected systems while motivating them to direct attention to their partners.

Leverage amount of information given In designing these virtual experiences, designers must be aware of the viewpoint which they are lending to users, and leverage the possibilities of that viewpoint to enhance the experience. In Lands of Fog, we used this custom viewpoint to leverage the amount of information provided to users. For example, users were able to barely see collaborative elements as they peeked through the foggy scenario. This subtle level of revelation was meant to entice users to continue discovering and moving around the scenario to find all of the hidden treasures. Lending partial information increases the naturalness of the experience, as users have to affront and interpret unknown situations just as we seek to complete information gaps in new experiences.

\section{Conclusions}

When designing full-body interaction experiences designers can develop systems with a first-person or a third-person paradigm (Parés \& Altimira, 2013) which can deeply influence how users experience the virtual environment and the outcomes of the interactive experience. While developing systems for children with ASD with the objective of promoting engagement and socialization, designers should take into account the strengths and limitations presented with both first and third person approaches.

With the first person approach, users have a 1:1 relation with the virtual environment, which leads to a more natural mapping and less cognitive load. 
We have seen that it is easier for users to understand the mappings of their activity with the digital world, avoiding having to go through a learning phase of how they are represented in the virtual environment typical of third-person configurations. Moreover, the lack of a virtual counterpart for the users allows for users to keep focusing physically to their peers when socializing, bringing more natural social behaviors than in third-person interaction.

In physical configurations where users must stay at a distance from the display, systems should provide clear feedback in terms of simple mappings or third person visualizations to help users understand their effects on the system. During collaborative experiences, the virtual counterpart of peers might help users to better understand their interaction space, actions, and intentions. In this case, researchers must be aware that players might look at the digital representation of their peer in lieu of face-to-face contact.

When creating collocated interfaces, designs aim for transparent or intuitive interfaces when referring to experiences where users have a very short adaptation time, helping users to use the interface nearly as in an unconscious manner (e.g. our experience with SIIMTA and Lands of Fog). Nonetheless, physical and virtual interfaces will always affect user's subjective experience of the system based on the selected viewpoint which is shared with the user. Our experience shows that when designing multi-user collocated experiences, designers must carefully analyze the implications of the interaction paradigm to understand how users perceive themselves, the environment, and other players during the interactive experience.

\section{References}

Ackermann, E. K. (2004). Constructing Knowledge and Transforming the World. A learning zone of one's own: Sharing representations and flow in collaborative learning environments, 15-37. Retrieved from http://web.media.mit.edu/\{ \}edith/publications/ 2004-Constructing $\left\{\backslash_{-}\right\}$Knowledge.pdf

Anderson, A., Locke, J., Kretzmann, M., Kasari, C., \& AIR-B Network. (2015). Social network analysis of children with autism spectrum disorder: Predictors of fragmentation and connectivity in elementary school classrooms. Autism. doi: 10.1177/1362361315603568

Antle, A. N. (2013). Research opportunities: Embodied child-computer interaction. International Journal of Child-Computer Interaction, 1(1), 30-36. doi: 10.1016/j.ijcci.2012.08.001

Antle, A. N., Corness, G., \& Droumeva, M. (2009, jan). What the body knows: Exploring the benefits of embodied metaphors in hybrid physical digital environments. Interacting with Computers, 21(1-2), 6675. Retrieved from http://www.sciencedirect.com/science/article/ pii/S0953543808000647 doi: 10.1016/j.intcom.2008.10.005

Association, A. P. (2013). Cautionary Statement for Forensic Use of DSM-5. In A. P. Association (Ed.), Diagnostic and statistical manual of mental 
disorders, 5th edition (5th ed.). Washington, DC: American Psychiatric Publishing, Inc. Retrieved from http://dsm.psychiatryonline.org// content . aspx?bookid=556\{\\&\}sectionid=41101754 doi: $10.1176 /$ appi .books.9780890425596.744053

Ayres, A. J., \& Tickle, L. (1980). Hyper-responsivity to Touch and Vestibular Stimuli as a Predictor of Positive Response to Sensory Integration Procedures by Autistic Children. Retrieved from http://journal.frontiersin .org/article/10.3389/fnint.2012.00124/abstract

Baron-Cohen, S. (2008). Autism and Asperger Syndrome. Oxford University Press. Retrieved from https://books.google.es/books/ about/Autism $\left\{\backslash_{-}\right\}$and $\left\{\backslash_{-}\right\}$Asperger $\left\{\backslash_{-}\right\}$Syndrome.html?id=DMR $-i X X A B Y_{w C}\{\backslash \&\}$ pgis $=1$

Bartoli, L., Corradi, C., Garzotto, F., \& Valoriani, M. (2013, jun). Exploring motion-based touchless games for autistic children's learning. In Proceedings of the 12th international conference on interaction design and children - idc '13 (pp. 102-111). New York, New York, USA: ACM Press. Retrieved from http://dl.acm.org/citation.cfm?id=2485760.2485774 doi: 10 $.1145 / 2485760.2485774$

Bekker, T., \& Sturm, J. (2009, jun). Stimulating physical and social activity through open-ended play. In Proceedings of the 8th international conference on interaction design and children - idc '09 (p. 309). New York, New York, USA: ACM Press. Retrieved from http://dl.acm.org/ citation. cfm?id=1551788.1551869 doi: 10.1145/1551788.1551869

Benson, J. B., \& Užgiris, I. Č. (1985). Effect of self-initiated locomotion on infant search activity. Developmental Psychology, 21(6), 923-931.

Bianchi-Berthouze, N., Kim, W. W., \& Patel, D. (2007). Does Body Movement Engage You More in Digital Game Play? and Why? In Affective computing and intelligent interaction (pp. 102-113). Berlin, Heidelberg: Springer Berlin Heidelberg. Retrieved from http://link.springer.com/10.1007/ 978-3-540-74889-2\{\_\}10 doi: 10.1007/978-3-540-74889-2_10

Borghi, A. M., \& Cimatti, F. (2010, mar). Embodied cognition and beyond: acting and sensing the body. Neuropsychologia, 48(3), 76373. Retrieved from http://www.sciencedirect.com/science/article/ pii/S0028393209004369 doi: 10.1016/j.neuropsychologia.2009.10.029

Casas, X., Herrera, G., Coma, I., \& Fernandez, M. (2012). A Kinect-Based Augmented Reality System for Individuals with Autism Spectrum Disorders. GRAPP/IVAPP , 440-446. Retrieved from http://www. scitepress.org/ DigitalLibrary/PublicationsDetail . aspx ID $=5$ pREawmgpko $=\{\backslash \&\} t=1$ doi: 978-989-8565-02-0

Cerbone, D. R. (2006). Understanding phenomenology. Retrieved from https://books.google.es/books/about/Understanding\{ -3phenomenology.html?id=q-7WAAAAMAAJ $\{\backslash \&\}$ pgis $=1$

Chen, W. (2012). Multitouch Tabletop Technology for People with Autism Spectrum Disorder: A Review of the Literature. Procedia Computer Science, 14, 198-207. Retrieved from http://www.sciencedirect.com/science/ 
article/pii/S1877050912007855 doi: 10.1016/j.procs.2012.10.023

Christensen, D. L., Bilder, D. A., Zahorodny, W., Pettygrove, S., Durkin, M. S., Fitzgerald, R. T., ... Yeargin-Allsopp, M. (2016, jan). Prevalence and Characteristics of Autism Spectrum Disorder Among 4-Year-Old Children in the Autism and Developmental Disabilities Monitoring Network. Journal of developmental and behavioral pediatrics : JDBP, 37(1), 1-8. Retrieved from http://www.ncbi.nlm.nih.gov/pubmed/26651088

Dalsgaard, P., \& Dindler, C. (2014, apr). Between theory and practice. In Proceedings of the 32nd annual acm conference on human factors in computing systems - chi '14 (pp. 1635-1644). New York, New York, USA: ACM Press. Retrieved from http://dl.acm.org/citation.cfm?id=2611528 . 2557342 doi: $10.1145 / 2556288.2557342$

De Jaegher, H. (2013, jan). Embodiment and sense-making in autism. Frontiers in integrative neuroscience, 7, 15. Retrieved from http://www.pubmedcentral.nih.gov/articlerender.fcgi?artid= 3607806\{\\&\}tool=pmcentrez $\{\backslash \&\}$ rendertype=abstract doi: 10.3389/fnint.2013.00015

Dillenbourg, P., \& Evans, M. (2011, dec). Interactive tabletops in education. International Journal of Computer-Supported Collaborative Learning, 6(4), 491-514. Retrieved from http://link.springer.com/10.1007/ s11412-011-9127-7 doi: 10.1007/s11412-011-9127-7

Donnellan, A. M., Hill, D. A., \& Leary, M. R. (2013). Rethinking autism: implications of sensory and movement differences for understanding and support. Frontiers in Integrative Neuroscience, 6(January), 1-11. Retrieved from http://journal.frontiersin.org/article/10.3389/fnint. 2012 .00124/abstract doi: 10.3389/fnint.2012.00124

Dourish, P. (2004). Where the Action is: The Foundations of Embodied Interaction. Retrieved from https://books.google.es/books/about/ Where $\left\{\backslash_{-}\right\}$the $\left\{\backslash_{-}\right\}$Action $\left\{\backslash_{-}\right\}$is.html?id=DCIy2zxrCqcC $\{\backslash \&\}$ pgis $=1$

Eason, L. J., White, M. J., \& Newsom, C. (1982, jan). Generalized reduction of self-stimulatory behavior: An effect of teaching appropriate play to autistic children. Analysis and Intervention in Developmental Disabilities, 2(2-3), 157-169. Retrieved from http://linkinghub.elsevier.com/retrieve/ pii/0270468482900167 doi: 10.1016/0270-4684(82)90016-7

Ellis, S. (1991, jan). Nature and origins of virtual environments: a bibliographical essay. Computing Systems in Engineering, 2(4), 321347. Retrieved from http://linkinghub.elsevier.com/retrieve/pii/ 095605219190001L doi: 10.1016/0956-0521(91)90001-L

Frischen, A., Bayliss, A. P., \& Tipper, S. P. (2007). Gaze cueing of attention: Visual attention, social cognition, and individual differences. Psychological Bulletin, 133(4), 694-724. Retrieved from http://doi.apa.org/getdoi .cfm?doi=10.1037/0033-2909.133.4.694 doi: 10.1037/0033-2909.133.4 .694

Frith, U., \& De Vignemont, F. (2005, dec). Egocentrism, allocentrism, and Asperger syndrome (Vol. 14) (No. 4). Retrieved from http://www.ncbi 
.nlm.nih.gov/pubmed/15996486 doi: 10.1016/j.concog.2005.04.006

Gabriels, R. L., Cuccaro, M. L., Hill, D. E., Ivers, B. J., \& Goldson, E. (2005, jan). Repetitive behaviors in autism: relationships with associated clinical features. Research in developmental disabilities, 26(2), 16981. Retrieved from http://www.sciencedirect.com/science/article/ $\mathrm{pii/S0891422204000897}$

Gillberg, C., \& Kadesjö, B. (2003, jan). Why bother about clumsiness? The implications of having developmental coordination disorder (DCD). Neural plasticity, 10(1-2), 59-68. Retrieved from http://www . pubmedcentral.nih.gov/articlerender.fcgi?artid= $2565425\{\backslash \&\}$ tool=pmcentrez $\{\backslash \&\}$ rendertype=abstract

Grandhi, S. A., Joue, G., \& Mittelberg, I. (2011, may). Understanding naturalness and intuitiveness in gesture production. In Proceedings of the 2011 annual conference on human factors in computing systems - chi '11 (p. 821). New York, New York, USA: ACM Press. Retrieved from http://dl.acm.org/citation.cfm?id=1978942.1979061 doi: 10.1145/ 1978942.1979061

Grudin, J. (1990, mar). The computer reaches out: the historical continuity of interface design. In Proceedings of the sigchi conference on human factors in computing systems empowering people - chi '90 (pp. 261-268). New York, New York, USA: ACM Press. Retrieved from http://dl.acm.org/ citation. $c f m$ ? id $=97243.97284$

Harrison, S., Tatar, D., \& Sengers, P. (2007). The Three Paradigms of HCI. In Chi (pp. 1-21). San Jose, USA.

Hartson, Rex, P. P. (2012). The UX Book - Process and guidlines for ensuring a quality of user experience (Vol. 1). Morgan Kaufmann.

Heim, S. (2008). The Resonant Interface: HCI Foundations for Interaction Design. Pearson/Addison Wesley. Retrieved from http://www.loc.gov/ catdir/toc/ecip079/2007003079.html

Hoberman, P., Pares, N., \& Pares, R. (1999, jan). El ball del fanalet or lightpools.

Horn, M. S. (2013). The role of cultural forms in tangible interaction design. In Proceedings of the 7th international conference on tangible, embedded and embodied interaction - tei '13 (p. 117). New York, New York, USA: ACM Press. Retrieved from http://dl.acm.org/citation. cfm?doid=2460625 .2460643 doi: $10.1145 / 2460625.2460643$

Hornecker, E., \& Buur, J. (2006, apr). Getting a grip on tangible interaction. In Proceedings of the sigchi conference on human factors in computing systems - chi '06 (p. 437). New York, New York, USA: ACM Press. Retrieved from http://dl.acm.org/citation.cfm?id=1124772.1124838 doi: 10 $.1145 / 1124772.1124838$

Howison, M., Trninic, D., Reinholz, D., \& Abrahamson, D. (2011). The Mathematical Imagery Trainer: from embodied interaction to conceptual learning. In Proceedings of the annual meeting of chi: Acm conference on human factors in computing systems (chi 2011) (pp. 1989 - 1998). ACM. 
Johnson, C. P., \& Myers, S. M. (2007, nov). Identification and evaluation of children with autism spectrum disorders. Pediatrics, 120(5), 1183-215. Retrieved from http://pediatrics.aappublications.org/content/120/ $5 / 1183$

Keay-Bright, W. (2007, jan). The Reactive Colours Project: Demonstrating Participatory and Collaborative Design Methods for the Creation of Software for Autistic Children. Retrieved from http://repository.uwic.ac.uk/ dspace/handle/10369/158

Kim, Y. S., Leventhal, B. L., Koh, Y.-J., Fombonne, E., Laska, E., Lim, E.-C., .. Grinker, R. R. (2011, sep). Prevalence of Autism Spectrum Disorders in a Total Population Sample. American Journal of Psychiatry, 168(9), 904-912. Retrieved from http://www.ncbi.nlm.nih.gov/ pubmed/21558103http://psychiatryonline.org/doi/abs/10.1176/ appi.ajp.2011.10101532 doi: 10.1176/appi.ajp.2011.10101532

Klatzky, R. (1998). Allocentric and egocentric spatial representations: Definitions, distinctions, and interconnections. Spatial cognition - An interdisciplinary approach to representation and processing of spatial knowledge(September 1997), 1-17. Retrieved from http://link. springer.com/10.1007/3-540-69342-4\{\_\}1http:// link. springer.com/chapter/10.1007/3-540-69342-4\{\_\}1 doi: 10.1007/3-540-69342-4_1

Koegel, R. I., \& Frea, W. D. (1993). Treatment of social behavior in autism through the modification of pivotal social skills. Journal of Applied Behavior Analysis, 26 (3), 1297760. Retrieved from http://www.pubmedcentral .gov/articlerender.fcgi?artid=1297760 doi: 10.1901/jaba.1993.26 $-369$

Krueger, M. W., Gionfriddo, T., \& Hinrichsen, K. (1985, apr). VIDEOPLACE - An Artificial Reality. In Chi'85 proceedings of the sigchi conference on human factors in computing systems (Vol. 16, pp. 35-40). ACM. Retrieved from http://dl.acm.org/citation.cfm?id=1165385.317463 doi: 10 $.1145 / 317456.317463$

Lane, A. E., Young, R. L., Baker, A. E. Z., \& Angley, M. T. (2010, jan). Sensory Processing Subtypes in Autism: Association with Adaptive Behavior. Journal of Autism and Developmental Disorders, 40(1), 112-122. Retrieved from http://link.springer.com/10.1007/s10803-009-0840-2 doi: 10.1007/s10803-009-0840-2

Lindblom, J., \& Alenljung, B. (2015). Socially Embodied HumanRobot Interaction. In (pp. 169-190). Retrieved from http:// services.igi-global. com/resolvedoi/resolve. aspx?doi=10.4018/ 978-1-4666-7278-9.ch007 doi: 10.4018/978-1-4666-7278-9.ch007

Liu, T. (2013, feb). Sensory processing and motor skill performance in elementary school children with autism spectrum disorder. Perceptual and motor skills, 116(1), 197-209. Retrieved from http://www.ncbi.nlm.nih.gov/ pubmed/23829146

MacDonald, M., Lord, C., \& Ulrich, D. A. (2014, apr). Motor skills and cali- 
brated autism severity in young children with autism spectrum disorder. Adapted physical activity quarterly : APAQ, 31(2), 95-105. Retrieved from http://www.ncbi.nlm.nih.gov/pubmed/24762385

Mora-Guiard, J., Crowell, C., Pares, N., \& Heaton, P. (2016a). Lands of Fog: Helping Children with Autism in Social Interaction through a Full-Body Interactive Experience. In Proceedings of the the 15th international conference on interaction design and children - idc '16 (pp. 262-274). New York, New York, USA: ACM Press. Retrieved from http://dl.acm.org/ citation. cfm?doid=2930674. 2930695 doi: $10.1145 / 2930674.2930695$

Mora-Guiard, J., Crowell, C., Pares, N., \& Heaton, P. (2016b, oct). Sparking social initiation behaviors in children with Autism through fullbody Interaction. International Journal of Child-Computer Interaction. Retrieved from http://linkinghub.elsevier.com/retrieve/pii/ S2212868916300150 doi: 10.1016/j.ijcci.2016.10.006

Nielsen, M., Störring, M., Moeslund, T. B., \& Granum, E. (2004). A Procedure for Developing Intuitive and Ergonomic Gesture Interfaces for HCI. In Gesture-based communication in human-computer interaction (pp. 409420). Retrieved from http://link.springer.com/10.1007/978-3-540 -24598-8\{\_\}38 doi: 10.1007/978-3-540-24598-8_38

Ortega, D. H., Cibrian, F. L., \& Tentori, M. (2015). BendableSound. In Proceedings of the 17th international acm sigaccess conference on computers 83 accessibility - assets '15 (pp. 315-316). New York, New York, USA: ACM Press. Retrieved from http://dl.acm.org/citation.cfm?doid= 2700648.2811355 doi: 10.1145/2700648.2811355

Parés, N., \& Altimira, D. (2013). Analyzing the Adequacy of Interaction Paradigms in Artificial Reality Experiences. HumanComputer Interaction, 28(2), 77-114. doi: 10.1080/07370024.2012.688469

Parés, N., Masri, P., van Wolferen, G., \& Creed, C. (n.d., jan). Achieving dialogue with children with severe autism in an adaptive multisensory interaction: the "MEDIAte" project. IEEE transactions on visualization and computer graphics, 11(6), 734-43. Retrieved from http:// www.ncbi.nlm.nih.gov/pubmed/16270865

Parés, N., \& Parés, R. (2006, oct). Towards a Model for a Virtual Reality Experience: The Virtual Subjectiveness. Presence: Teleoperators and Virtual Environments, 15(5), 524-538. Retrieved from http:// www.mitpress journals.org/doi/abs/10.1162/pres.15.5.524 doi: 10 $.1162 /$ pres.15.5.524

Peeters, G., Grobben, G., Hendrickx, A., Van den Eede, S., \& Verlinden, K. (2003, apr). Self-other and third-person categorization in normal and autistic children. Developmental Science, 6(2), 166-172. Retrieved from http://doi.wiley.com/10.1111/1467-7687.00267 doi: 10.1111/ 1467-7687.00267

Porayska-Pomsta, K., Frauenberger, C., Pain, H., Rajendran, G., Smith, T., Menzies, R., ... Lemon, O. (2011, may). Developing technology for autism: an interdisciplinary approach. Per- 
sonal and Ubiquitous Computing, 16(2), 117-127. Retrieved from http://www.research.ed.ac.uk/portal/en/publications/ developing-technology-for-autism-an-interdisciplinary -approach (d000f4e7-a65b-40f0-aaaa-d48164d33c88) .html

Rajendran, G., Law, A. S., Logie, R. H., Van Der Meulen, M., Fraser, D., \& Corley, M. (2011, nov). Investigating multitasking in high-functioning adolescents with autism spectrum disorders using the virtual errands task. Journal of Autism and Developmental Disorders, 41(11), 1445-1454. Retrieved from http://link.springer.com/10.1007/s10803-010-1151-3 doi: 10.1007/s10803-010-1151-3

Revelle, G. (2013, jan). Applying developmental theory and research to the creation of educational games. New directions for child and adolescent development, 2013(139), 31-40. Retrieved from http://www.ncbi.nlm .nih.gov/pubmed/23483691 doi: 10.1002/cad.20029

Ringland, K. E., Zalapa, R., Neal, M., Escobedo, L., Tentori, M., \& Hayes, G. R. (2014). SensoryPaint. In Proceedings of the 2014 acm international joint conference on pervasive and ubiquitous computing - ubicomp '14 adjunct (pp. 873-884). New York, New York, USA: ACM Press. Retrieved from http://dl.acm.org/citation. cfm?doid=2632048.2632065 doi: $10.1145 / 2632048.2632065$

Rowland, C. M., \& Schweigert, P. D. (2009, apr). Object lessons: How children with autism spectrum disorders use objects to interact with the physical and social environments. Research in Autism Spectrum Disorders, 3(2), 517-527. Retrieved from http://www.sciencedirect.com/ science/article/pii/S1750946708001311

Stephenson, J., \& Limbrick, L. (2015, dec). A Review of the Use of TouchScreen Mobile Devices by People with Developmental Disabilities. Journal of Autism and Developmental Disorders, 45(12), 3777-3791. Retrieved from http://link.springer.com/10.1007/s10803-013-1878-8 doi: 10 .1007/s10803-013-1878-8

Vazquez, V., Cardenas, C., Cibrian, F. L., \& Tentori, M. (2016). Designing a Musical Fabric-Based Surface to Encourage Children with Autism to Practice Motor Movements. In Proceedings of the 6th mexican conference on human-computer interaction - mexihc'16 (pp. 1-4). New York, New York, USA: ACM Press. Retrieved from http://dl.acm.org/citation .cfm?doid=2967175.2967384 doi: 10.1145/2967175.2967384

Vogeley, K., May, M., Ritzl, A., Falkai, P., Zilles, K., \& Fink, G. R. (2004a, jun). Neural correlates of first-person perspective as one constituent of human self-consciousness. Journal of cognitive neuroscience, 16(5), 817-27. Retrieved from http://www.ncbi.nlm.nih.gov/pubmed/15200709 doi: 10.1162/089892904970799

Vogeley, K., May, M., Ritzl, A., Falkai, P., Zilles, K., \& Fink, G. R. (2004b, jun). Neural Correlates of First-Person Perspective as One Constituent of Human Self-Consciousness. Journal of Cognitive Neuroscience, 16(5), 817-827. Retrieved from http://www.mitpressjournals.org/doi/abs/ 
10.1162/089892904970799 doi: 10.1162/089892904970799

Wertsch, J. V. (1985). Vygotsky and the social formation of mind.

Williams, E. (2003, dec). A comparative review of early forms of object-directed play and parent-infant play in typical infants and young children with autism. Autism : the international journal of research and practice, 7(4), $361-77$. 